\title{
Diversidade de Scarabaeinae (Coleoptera, Scarabaeidae) coletados com armadilha de interceptação de vôo no Refúgio Ecológico Charles Darwin, Igarassu-PE, Brasil
}

\author{
Cristiane Maria Queiroz da Costa ${ }^{1}$, Fernando Augusto Barbosa Silva ${ }^{1,2}$, Ângela Isidro de Farias ${ }^{2}$ \\ \& Rita de Cássia de Moura ${ }^{1}$
}

${ }^{1}$ Departamento de Biologia-ICB. Universidade de Pernambuco. Rua Arnóbio Marques 310, 50100-130 Recife-PE, Brasil.

${ }^{2}$ Programa de Pós-graduação em Biologia Animal, Departamento de Zoologia-UFPE, Av. Prof. Moraes Rego s/n, Cidade Universitária 50.670-420

Recife-PE, cristianemqc@yahoo.com.br; fernandoabsilva@yahoo.com.br; amif33@ hotmail.com; rita_upe@yahoo.com.br

\begin{abstract}
Diversity of Scarabaeinae (Coleoptera, Scarabaeidae) collected with flight intercept trap in the Charles Darwin Ecologic Refuge, Igarassu-PE, Brazil. The objective of this work was to study richness, abundance, diversity and equitability of the species of Scarabaeinae, in two environments in the Charles Darwin Ecology Refuge (CDER), Pernambuco. Sampling was carried with flight intercept trap from April through June 2007, totaling six samples. 4576 scarabaeinaes, belonging to 35 species, 15 genera and six tribes were captured. Seven new species records were found for Pernambuco, whose known fauna increased from 26 to 33 species. The tribes recorded were Canthonini, Ateuchini, Coprini, Phanaeinae, Eurystenini and Onthophagini. The better represented genera were: Dichotomius with $84,6 \%$ of the beetles collected, followed by Canthidium with 7,62\% and Canthon with 2,48\%. Dichotomius aff. sericeus was the most abundant species with 3889 individuals. 17 species were sampled in open areas, with 11 of them restricted to this habitat, while 24 were recorded within the forest, 18 of them found only in this habitat. Most individuals captured in the forest belong to $D$. aff. sericeus, while in open areas no specimen of this species was collected. The estimators indicate a maximum richness of 21 species for the open environment and 32 species for the forest. The species accumulation curve, based on average richness values for the two environments at CDER, did not show tendency to assintote.
\end{abstract}

KEYWORDS. Community ecology; Atlantic Forest; dung beetles.

RESUMO. Diversidade de Scarabaeinae (Coleoptera, Scarabaeidae) coletados com armadilha de interceptação de vôo no Refúgio Ecológico Charles Darwin, Igarassu-PE, Brasil. O objetivo deste trabalho foi estudar riqueza, abundância, diversidade e equitabilidade das espécies de Scarabaeinae em dois ambientes no Refúgio Ecológico Charles Darwin (RECD), Pernambuco. As coletas foram realizadas com armadilha de interceptação de vôo entre os meses de Abril e Junho de 2007, totalizando seis coletas. Foram capturados 4576 escarabeíneos, pertencentes a 35 espécies, 15 gêneros e seis tribos. Sete novos registros de espécies foram feitos para Pernambuco, passando de 26 para 33 espécies. As tribos registradas foram Canthonini, Ateuchini, Coprini, Phanaeini, Eurystenini e Onthophagini. Os gêneros melhor representados foram: Dichotomius com $84,6 \%$ dos besouros coletados, seguido por Canthidium com 7,62\% e Canthon com 2,48\%. A espécie Dichotomius aff. sericeus foi a mais abundante com 3889 indivíduos. Em todo o estudo foram verificadas 17 espécies para a área aberta, sendo 11 restritas a esse ambiente, enquanto para mata foram registradas 24 , sendo 18 espécies restritas. Grande parte dos indivíduos capturados na mata foi da espécie $D$. aff. sericeus, enquanto no ambiente aberto nenhum indivíduo desta espécie foi coletado. Os estimadores indicaram uma estimativa máxima de riqueza de 21 espécies para o ambiente aberto e 32 espécies para o ambiente de mata. A curva de acumulação de espécies baseada em valores de riqueza observada média para os dois ambientes do RECD, não apresentou tendência a assíntota.

PALAVRAS-CHAVE. Ecologia de Comunidade; Mata atlântica; rola-bosta.

Modificação e fragmentação de habitats compreendem os dois tipos mais comuns de conversão da paisagem. A fragmentação envolve a reconfiguração de um habitat em pequenos e isolados "retalhos" dentro de uma matriz de habitat modificado (Nichols et al. 2007). Áreas abertas criadas para agricultura, desenvolvimento urbano e outros propósitos, nas florestas naturais, originam paisagens fragmentadas e condições microclimáticas distintas (maior intensidade luminosa, incidência de ventos e amplitude térmica) devido à retirada da cobertura florestal (Dajoz 1978; Gimenes \& Anjos 2003; Rodrigues \& Marchini 2000). Essas alterações microclimáticas provocam um declínio na biodiversidade (Primack \& Rodrigues 2001).
A Floresta Atlântica brasileira foi identificada por Myers et al. (2000) como um dos pontos mais críticos "hottest hotspots" para ações prioritárias de conservação. No Nordeste brasileiro existem apenas $2 \%$ da floresta original, dispersa na forma de pequenos fragmentos circundados por áreas abertas ou localizados dentro de áreas urbanas (Ranta et al. 1998). Em Pernambuco, os remanescentes florestais da Mata Atlântica ocupam uma área de 1,54\% $\left(1.524 \mathrm{~km}^{2}\right)$ do estado (Tabarelli et al. 2006).

Para se estimar a diversidade biológica de uma região é importante a escolha de um grupo indicador, o qual será capaz de informar o efeito de fatores ambientais, sejam eles naturais ou antropogênicos, na comunidade local. De modo geral, 
sugere-se a utilização de vários grupos para monitoramento da biodiversidade de insetos em fragmentos florestais: borboletas, principalmente Nymphalidae (Lepidoptera); besouros, principalmente pertencentes às famílias Scarabaeidae e Carabidae (Coleoptera); formigas e abelhas, da subfamília Euglossinae (Hymenoptera) e cupins (Isoptera) (Brown 1991, 1997; Brown \& Freitas 2000; Thomazini \& Thomazini 2000; Freitas et al. 2006).

A ordem Coleoptera é a mais representativa da classe Insecta com 357.899 espécies descritas (40\% do total de insetos) (Lawrence \& Britton 1991). Segundo Costa (2000), para a região neotropical estão registradas 127 famílias, 6.703 gêneros e 72.476 espécies de besouros. Dessas famílias, Scarabaeidae se destaca por apresentar grande irradiação adaptativa e abranger cerca de 25.000 espécies, compreendidas em sete subfamílias (Lawrence \& Newton 1995). Dentre estas subfamílias, Scarabaeinae é uma das mais bem conhecidas em termos taxonômicos e funcionais, compreendendo 12 tribos, 234 gêneros e 6.000 espécies distribuídas em todo o mundo. A região neotropical possui registro de aproximadamente nove tribos, 70 gêneros e 1.250 espécies (Hanski \& Cambefort 1991). No Brasil, foram registradas 618 espécies incluídas em 49 gêneros (Vaz-de-Mello 2000), destas, 149 espécies foram verificadas para o Nordeste e 26 para Pernambuco (Vaz-deMello 2000; Silva et al. 2007).

Os escarabeíneos, usualmente chamados de "rola-bosta", possuem o hábito característico de processar matéria orgânica em decomposição, fabricando uma bola de alimento que pode ser rolada ou enterrada, servindo como substrato para a oviposição e futuro alimento de suas larvas. Esse comportamento contribui para a ciclagem de nitrogênio e dos outros nutrientes no ecossistema (Halffter \& Matthews 1966).

As comunidades de Scarabaeinae respondem fortemente a distúrbios ambientais, naturais ou antropogênicos, através de alterações na riqueza de espécies, distribuição de abundância, composição específica ou na estrutura de guildas (Andresen 2005; Gardner et al. 2008; Halffter \& Favila 1993; Spector \& Ayzama 2003). A cobertura florestal representa um elemento determinante da estrutura e diversidade da fauna destes besouros (Halffter \& Arellano 2002). Nos trópicos, geralmente, áreas abertas ou de campo possuem menor diversidade de espécies de Scarabaeinae que as áreas florestais (Halffter 1991; Howden \& Nealis 1975; Klein 1989). Entretanto, em áreas abertas há ocorrência de espécies (heliófilas) que não são encontradas dentro da floresta (Howden \& Nealis 1975).

Os levantamentos de Scarabaeinae realizados no Brasil, em sua maioria, foram feitos nas regiões Sul e Sudeste (Vazde-Mello 2000), utilizando armadilhas de solo como método de coleta. Entretanto, alguns levantamentos de escarabeíneos têm sido feitos no Nordeste nesses últimos anos (Endres et al. 2007; Hernández 2005; 2007; Lopes et al. 2006; Silva et al. 2007); em Pernambuco, num estudo realizado em um ambiente associado a ecossistema de Brejo de Altitude foram registradas 21 espécies (Silva et al. 2007). Contudo, para a região Nordeste nenhum trabalho foi realizado utilizando como método de captura armadilhas de interceptação de vôo.

Este estudo teve como objetivo analisar abundância, riqueza, diversidade e equitabilidade das espécies de Scarabaeinae, coletadas com armadilha de interceptação de vôo, em dois ambientes distintos, no Refúgio Ecológico Charles Darwin (RECD), Igarassu, Pernambuco.

\section{MATERIALE MÉTODOS}

O estudo foi desenvolvido no Refúgio Ecológico Charles Darwin (07² $38^{\prime} 37^{\prime \prime} \mathrm{S} ; 34^{\circ} 57^{\prime} 25^{\prime}$ 'W), uma reserva de Mata Attântica (RPPN) com 60 hectares, localizada no município de Igarassu, litoral Norte do Estado de Pernambuco. A área é cortada pelo rio Jacoca, também chamado de Tabatinga, que é perene e faz parte da bacia hidrográfica do rio Botafogo. A vegetação é típica da Zona da Mata Pernambucana, uma Formação Florestal Perenifólia Latifoliada Higrófila Costeira (Santiago \& Barros 2003). O clima do local é do tipo Tropical quente e úmido, com chuvas de outono/inverno, do tipo As' na classificação climática de Koppen. A temperatura média anual é de $25^{\circ} \mathrm{C}$ e a pluviosidade média anual está em torno dos $2000 \mathrm{~mm}$.

Os besouros foram coletados em dois ambientes dentro do RECD. O primeiro corresponde a um local de mata, formada por um estrato arbóreo variando entre 8 e $15 \mathrm{~m}$ de altura e um estrato herbáceo-arbustivo, e o segundo local corresponde a um ambiente aberto com predominância de gramíneas.

Foram realizadas seis coletas, com intervalos de 15 dias, nos meses de Abril, Maio e Junho de 2007. As armadilhas ficaram no campo todo o tempo de realização do estudo, sendo que a cada duas semanas eram coletados os animais capturados e a solução fixadora era reposta, quando necessário. Cada ambiente recebeu três armadilhas, em diferentes pontos, com distância mínima de $80 \mathrm{~m}$ entre elas.

A armadilha consistiu de uma tela de $1 \mathrm{~m}$ de altura por $2 \mathrm{~m}$ de comprimento, dois canos de PVC de 1 polegada com 1,5 m de comprimento, fios de náilon e seis recipientes plásticos de 3 litros, preenchidos com água, detergente e formol a $4 \%$, para a fixação dos escarabeíneos.

Após a captura, o material foi levado ao Laboratório de Biodiversidade e Genética de Insetos - UPE. As espécies foram identificadas pelo doutorando Fernando Silva (UFLA - MG) e pelo Dr. Fernando Z. Vaz-de-Mello (UFMT - MT).

A análise dos resultados foi feita a partir de estudo da abundância relativa dos indivíduos, bem como da riqueza, da diversidade e da equitabilidade das espécies. A abundância foi analisada conforme critérios sugeridos por Colwell (2005), em que espécies com abundância de um a dez indivíduos são consideradas "raras", dentre estas, as que possuem apenas um único indivíduo são tidas como "singleton" e com dois indivíduos "doubleton"; as espécies com abundância acima de dez indivíduos são ditas "comuns". A diversidade foi avaliada através do índice de Shannon na base exponencial, enquanto a equitabilidade foi medida pelo índice de Camargo (Kenney \& Krebs 2000). Para estimativa do número de espécies presentes nos ambientes estudados foram utilizados quatro 
estimadores não-paramétricos: Chao "1", Jackknife "1" e "2" que se baseiam em dados de abundância, utilizando a relação entre o número de "singletons" e "doubletons" para as estimativas de riqueza. E o estimador "Chao 2", que é baseado em incidência e utiliza o número de "uniques" (espécies encontradas em somente uma amostra) e "duplicates" (espécies encontradas em somente duas amostras) para as estimativas de riqueza (Colwell 2005). A curva de acumulação de espécies utilizada neste trabalho foi a de Michaelis-Menten, construída através de 100 randomizações. Os cálculos das estimativas de riqueza foram realizados através do programa EstimateS 7.5 (Colwell 2005) e os gráficos destas análises foram produzidos através do programa Statistica 6.0 (Statsoft 1998).

\section{RESULTADOS EDISCUSSÃO}

Foram coletados 4.576 Scarabaeinae, pertencentes a 35 espécies, 15 gêneros e seis tribos (Tab. I). Dos táxons coletados, sete espécies (Anomiopus nigricans Westwood, 1842, Canthon nigripennis Lansberge, 1874, C. septemmaculatus histrio (Serville, 1828), Coprophanaeus dardanus (MacLeay, 1819), C. ensifer (Germar, 1824), Dichotomius geminatus (Arrow, 1913) e Trichillum externepunctatum Borre, 1886) são novos registros para o estado de Pernambuco. O número de espécies atualmente registradas para Pernambuco passa de 26 (Silva et al. 2007) para 33 espécies. Segundo os trabalhos publicados com levantamento faunístico para região Nordeste com registro de espécies de Scarabaeinae, foram identificadas a nível específico até o momento 40 espécies nesta região, entretanto, aqui não foram computados os dados referentes aos trabalhos de

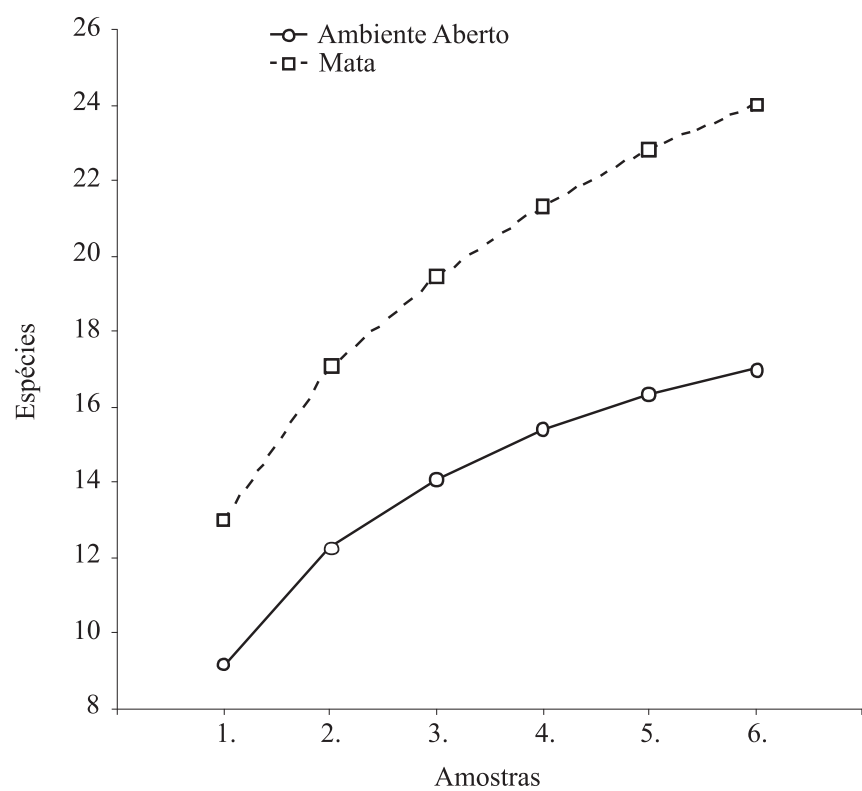

Fig. 1. Curva de acumulação de espécies de Scarabaeinae para os dois ambientes do Refúgio Ecológico Charles Darwin, em relação ao esforço amostral. A curva foi construída a partir de dados da riqueza observada média (Sobs). sistemática com espécies coletadas nestes locais (Tab. II). Algumas espécies registradas no presente trabalho também foram observadas em diferentes ambientes e ecossistemas da região nordeste do Brasil e localidades próximas (Tab. III).

Com relação às tribos registradas para este trabalho, Canthonini e Ateuchini foram as melhores representadas com 12 e 10 espécies respectivamente, seguidas por Coprini (5 espécies), Phanaeini (5), Eurystenini (2) e Onthophagini (1). A estrutura taxonômica das comunidades do RECD reflete parcialmente a estrutura observada para Região Neotropical,

Tabela I. Número de indivíduos por espécie e tribo de Scarabaeinae (Coleoptera, Scarabaeidae), coletados em armadilhas de interceptação de vôo, em ambientes de área aberta e mata, entre os meses de Abril e Junho de 2007, no Refúgio Ecológico Charles Darwin, Igarassu-PE.

\begin{tabular}{|c|c|c|c|}
\hline Tribo/espécie & Mata & $\begin{array}{c}\text { Área } \\
\text { aberta }\end{array}$ & Total \\
\hline \multicolumn{4}{|l|}{ Ateuchini } \\
\hline Anomiopus nigricans Westwood, 1842 & 0 & 24 & 24 \\
\hline Ateuchus sp.1 & 51 & 0 & 51 \\
\hline Ateuchus sp.2 & 13 & 0 & 13 \\
\hline Ateuchus sp. 3 & 0 & 2 & 2 \\
\hline Canthidium humerale (Germar, 1813) & 0 & 18 & 18 \\
\hline Canthidium sp. 1 & 313 & 3 & 316 \\
\hline Canthidium sp.2 & 1 & 11 & 12 \\
\hline Canthidium (Eucanthidium) sp.3 & 2 & 2 & 4 \\
\hline Canthidium sp.4 & 0 & 2 & 2 \\
\hline Uroxys sp. & 23 & 0 & 23 \\
\hline \multicolumn{4}{|l|}{ Canthonini } \\
\hline Canthon chalybaeus Blanchard, 1843 & 0 & 8 & 8 \\
\hline Canthon aff. juvencus Harold, 1868 & 2 & 4 & 6 \\
\hline Canthon lituratus (Germar, 1824) & 0 & 1 & 1 \\
\hline Canthon mutabilis Lucas, 1857 & 0 & 37 & 37 \\
\hline Canthon nigripennis Lansberge, 1874 & 38 & 0 & 38 \\
\hline Canthon septemmaculatus histrio (Serville, 1828) & 1 & 0 & 1 \\
\hline Canthon (Glaphyrocanthon) sp.1 & 0 & 24 & 24 \\
\hline Canthonella sp. & 11 & 0 & 11 \\
\hline Deltochilum sp.1 & 6 & 2 & 8 \\
\hline Deltochilum sp. 2 & 29 & 0 & 29 \\
\hline Pseudocanthon xanthurum (Blanchard, 1843) & 0 & 10 & 10 \\
\hline Sylvicanthon sp. & 3 & 0 & 3 \\
\hline \multicolumn{4}{|l|}{ Coprini } \\
\hline Dichotomius geminatus (Arrow, 1913) & 0 & 6 & 6 \\
\hline Dichotomius semisquamosus (Curtis, 1845) & 4 & 0 & 4 \\
\hline Dichotomius aff. sericeus (Harold, 1867) & 3889 & 0 & 3889 \\
\hline Ontherus aphodioides (Burmeister, 1874) & 1 & 0 & 1 \\
\hline Trichillum externepunctatum Borre, 1886 & 2 & 0 & 2 \\
\hline \multicolumn{4}{|l|}{ Eurysternini } \\
\hline Eurysternus caribaeus (Herbst, 1789) & 8 & 0 & 8 \\
\hline Eurysternus aff. hirtellus Dalman, 1824 & 1 & 0 & 1 \\
\hline \multicolumn{4}{|l|}{ Onthophagini } \\
\hline Onthophagus aff. hirculus Mannerheim, 1829 & 0 & 2 & 2 \\
\hline \multicolumn{4}{|l|}{ Phanaeini } \\
\hline Coprophanaeus dardanus (MacLeay, 1819) & 2 & 0 & 2 \\
\hline Coprophanaeus ensifer (Germar, 1824) & 1 & 0 & 1 \\
\hline Coprophanaeus cyanescens (d'Olsoufieff, 1924) & 2 & 3 & 5 \\
\hline Coprophanaeus pertyi (d'Olsoufieff, 1924) & 1 & 0 & 1 \\
\hline Coprophanaeus (Metallophanaeus) sp.1 & 13 & 0 & 13 \\
\hline Total & 4417 & 159 & 4576 \\
\hline
\end{tabular}


Tabela II. Lista das espécies de Scarabaeinae registradas para a região Nordeste em trabalhos de levantamento faunístico até o ano de 2007. * = novos registros para o estado de Pernambuco.

1 = presente trabalho; 2 = Endres et al. (2005); $3=$ Endres et al . (2007); 4 = Hernández (2005); 5 = Hernández (2007); 6 = Lopes et al. (2006); 7 = Silva et al. (2007).

\begin{tabular}{|c|c|c|}
\hline Espécies & Estado & Referência \\
\hline Anomiopus nigricans Westwood, $1842 *$ & $\mathrm{PE}$ & 1 \\
\hline Ateuchus carbonarius (Harold, 1868) & PB & 4,5 \\
\hline Canthidium humerale (Germar, 1813) & $\mathrm{BA}, \mathrm{PB}, \mathrm{PE}$ & $1,3,6,7$ \\
\hline Canthidium manni Arrow, 1913 & BA, PB & $3,4,5,6$ \\
\hline Canthon chalybaeus Blanchard, 1843 & PE & 1,7 \\
\hline Canthon lituratus (Germar, 1824) & $\mathrm{BA}, \mathrm{PB}, \mathrm{PE}$ & $1,3,4,6,7$ \\
\hline Canthon mutabilis Lucas, 1857 & PE & 1,7 \\
\hline Canthon nigripennis Lansberge, 1874* & $\mathrm{PB}, \mathrm{PE}$ & 1,3 \\
\hline $\begin{array}{l}\text { Canthon septemmaculatus histrio } \\
\text { (Serville, 1828)* }\end{array}$ & PE & 1 \\
\hline Canthon staigi (Pereira, 1953) & $\mathrm{PB}, \mathrm{PE}$ & 3,7 \\
\hline Coprophanaeus dardanus (MacLeay, 1819)* & * PE & 1 \\
\hline Coprophanaeus ensifer (Germar, 1824) * & $\mathrm{PB}, \mathrm{PE}$ & $1,2,3$ \\
\hline $\begin{array}{l}\text { Coprophanaeus cyanescens } \\
\text { (d'Olsoufieff, 1924) }\end{array}$ & $\mathrm{BA}, \mathrm{PB}, \mathrm{PE}$ & $1,6,7$ \\
\hline Coprophanaeus pertyi (d'Olsoufieff, 1924) & BA, PE & $1,5,6,7$ \\
\hline Deltochilum irroratum (Laporte, 1840) & PE & 7 \\
\hline Deltochilum pseudoicarus Balthasar, 1939 & PB & 3 \\
\hline Deltochilum verruciferum Felsche, 1911 B & $\mathrm{BA}, \mathrm{PB}, \mathrm{PE}$ & $4,5,6,7$ \\
\hline Dichotomius bos (Blanchard, 1843) & $\mathrm{PE}$ & 7 \\
\hline Dichotomius crinicollis (Germar,1824) & $\mathrm{PE}$ & 7 \\
\hline Dichotomius geminatus (Arrow, 1913)* B & $\mathrm{BA}, \mathrm{PB}, \mathrm{PE}$ & $1,3,4,5,6$ \\
\hline $\begin{array}{l}\text { Dichotomius machadoi Martínez e Pereira, } \\
1967 \text { (endêmica) }\end{array}$ & PE & 7 \\
\hline Dichotomius nisus (Olivier, 1789) & $\mathrm{PB}, \mathrm{PE}$ & $3,4,5,7$ \\
\hline Dichotomius semisquamosus (Curtis, 1845) & $\mathrm{PB}, \mathrm{PE}$ & $1,3,7$ \\
\hline Dichotomius sericeus (Harold, 1867) & $\mathrm{PB}, \mathrm{PE}$ & $1,3,7$ \\
\hline Diabroctis mimas mimas (Linnaeus, 1758) & BA, PE & 6,7 \\
\hline $\begin{array}{l}\text { Digitonthophagus gazella (Fabricius, 1787) } \\
\text { (espécie exótica) }\end{array}$ & BA, PE & 6,7 \\
\hline Eurysternus caribaeus (Herbst, 1789) & PE & 1,7 \\
\hline Eurysternus hirtellus Dalman, 1824 & $\mathrm{~PB}, \mathrm{PE}$ & $1,3,7$ \\
\hline $\begin{array}{l}\text { Malagoniella astyanax astyanax } \\
\text { (Olivier, 1789) }\end{array}$ & $\mathrm{PB}, \mathrm{PE}$ & $4,5,7$ \\
\hline Ontherus aphodioides (Burmeister, 1874) & $\mathrm{PE}$ & 1,7 \\
\hline Ontherus appendiculatus (Mannerhein, 1829 & $\mathrm{PE}$ & 7 \\
\hline Ontherus digitatus Harold, 1868 & PE & 5,7 \\
\hline Onthophagus hirculus Mannerheim, 1829 & $\mathrm{~PB}$ & 4,5 \\
\hline Onthophagus ranunculus Arrow, 1913 & BA & 6 \\
\hline Onthophagus rubrescens Blanchard, 1843 & BA & 6 \\
\hline $\begin{array}{l}\text { Pseudocanthon xanthurum } \\
\text { (Blanchard, 1843) }\end{array}$ & BA, PE & $1,6,7$ \\
\hline $\begin{array}{l}\text { Scybalocanthon cyanocephalum (Harold, } \\
\text { 1868) (identificação incerta) }\end{array}$ & $\mathrm{PE}$ & 7 \\
\hline $\begin{array}{l}\text { Sylvicanthon machadoi (Pereira e } \\
\text { Martínez, 1967) (endêmica) }\end{array}$ & $\mathrm{PE}$ & 7 \\
\hline Trichillum externepunctatum Borre, $1886^{*}$ B & $\mathrm{BA}, \mathrm{PB}, \mathrm{PE}$ & $1,3,6$ \\
\hline Uroxys corporaali Balthasar, 1940 & BA & 6 \\
\hline
\end{tabular}

se consideradas as tribos cuja distribuição geográfica inclui o estado de Pernambuco (Louzada \& Lopes 1997). As diferenças observadas entre a estrutura de Região Neotropical e do RECD está na posição invertida das tribos Eurysternini e
Onthophagini neste estudo. Silva et al. (2007), estudando ambiente associado a um ecossistema de Brejo de Altitude, em Caruaru - PE, também observou pequenas diferenças na estrutura taxonômica das tribos quando comparada a da Região Neotropical. Já Endres et al. (2007), estudando Mata Atlântica e Tabuleiro Nordestino encontrou estrutura semelhante a da Região Neotropical. Embora todas as tribos que ocorrem na região Nordeste tenham sido representadas nestes estudos, as proporções observadas entre estas tribos em diferentes locais podem variar de acordo com aspectos regionais. Louzada \& Lopes (1997) sugerem que estes desvios são possivelmente ligados a fatores biogeográficos ou ecológicos dos representantes das tribos em questão.

Os gêneros melhor representados foram: Dichotomius Hope, 1838 (3.899 indivíduos) com 84,6\% dos besouros coletados, seguido por Canthidium Erichson, 1847 (352 indivíduos) com 7,62\% e Canthon Hoffmannsegg, 1817 (115 indivíduos) com 2,48\%. Embora, o grande número de indivíduos registrados para o gênero Dichotomius deva-se em grande parte a abundância de $D$. aff. sericeus (Harold, 1867) $(\mathrm{N}=3889)$, o que corresponde a $84,37 \%$ de todos os indivíduos coletados neste estudo.

O fato do Refúgio Ecológico Charles Darwin encontrar-se em uma área com um processo contínuo de antropização, com conseqüente redução e isolamento dos fragmentos de mata, pode ter ocasionado uma mudança na distribuição das espécies assim como nas suas abundâncias relativas. O impacto ambiental sofrido por estes ecossistemas pode ter favorecido espécies oportunistas e mais tolerantes às mudanças na dinâmica da comunidade (Saunders et al.1991).

Escobar (1997) e Favila \& Halffter (1997), citam como conseqüência da fragmentação e redução das áreas de mata em regiões tropicais as mudanças na estrutura e funcionamento das comunidades, diminuição do fluxo gênico das populações, perda de hábitat com conseqüente redução do número de espécies, e a própria extinção de espécies mais vulneráveis. Dale et al. (1995) sugere que a sensibilidade dos Scarabaeinae a mudanças no hábitat é ocasionada principalmente pela baixa capacidade de dispersão da maioria das espécies, bem como pela dieta especializada de muitas espécies, baseada principalmente em excrementos de mamíferos.

Analisando a abundância das populações foram registradas para mata 15 espécies raras, sendo seis "singletons", cinco "doubletons" e quatro com abundância entre três e 10 indivíduos, nove espécies foram "comuns". Já no ambiente aberto foram registradas 12 espécies raras, sendo uma "singleton", cinco "doubletons" e seis com abundancia entre três e 10 indivíduos. Foram registradas cinco espécies consideradas comuns para esse ambiente (Tab. IV). Quando analisado o percentual das espécies raras e comuns, verificase que os dois ambientes apresentaram o mesmo padrão, isto é, as espécies raras foram predominantes. Esse resultado corrobora com os dados de Halffter (1991), onde o autor afirma que é comum encontrar nas florestas tropicais e ambientes associados, um grande número de indivíduos pertencentes a poucas espécies, enquanto grande parte das espécies é 
Tabela III. Espécies levantadas no presente trabalho com registro de ocorrência em outros estudos ecológicos realizados na região Nordeste e nas suas proximidades.

\begin{tabular}{|c|c|c|}
\hline Espécies & Ocorrência na região Nordeste e proximidades & Referência \\
\hline $\begin{array}{l}\text { Canthidium humerale } \\
\quad \text { (Germar, 1813) }\end{array}$ & Caatinga, tabuleiro e brejo de altitude & $\begin{array}{l}\text { Endres et al. 2007; Lopes et al. } \\
\quad \text { 2006; Silva et al. } 2007\end{array}$ \\
\hline $\begin{array}{l}\text { Canthon chalybaeus } \\
\text { Blanchard, } 1843\end{array}$ & Brejo de altitude associada a área de pastagem & Silva et al. 2007 \\
\hline $\begin{array}{l}\text { Canthon lituratus } \\
\text { (Germar, 1824) }\end{array}$ & $\begin{array}{l}\text { Caatinga; tabuleiro nordestino; brejo de altitude, Caruaru - PE; } \\
\text { área de pastagem na região do Delta do Rio Doce - ES }\end{array}$ & $\begin{array}{l}\text { Hernández 2005; Lopes et al. 2006; } \\
\text { Endres et al. 2007; Silva et al. 2007; } \\
\quad \text { Schiffler } \text { et al. } 2003\end{array}$ \\
\hline $\begin{array}{l}\text { Canthon mutabilis } \\
\text { Lucas, } 1857\end{array}$ & Brejo de altitude associada a área de pastagem & Silva et al. 2007 \\
\hline $\begin{array}{l}\text { Canthon nigripennis } \\
\text { Lansberge, } 1874\end{array}$ & Mata atlântica e tabuleiro nordestino & Endres et al. 2007 \\
\hline $\begin{array}{l}\text { Coprophanaeus dardanus } \\
\quad \text { (MacLeay, 1819) }\end{array}$ & $\begin{array}{l}\text { Encontrada em cultura de cacau, próximo a remanescente de } \\
\text { mata atlântica, bem como em área de restinga arbustiva }\end{array}$ & Schiffler et al. 2003 \\
\hline $\begin{array}{l}\text { Coprophanaeus ensifer } \\
\quad(\text { Germar, 1824) }\end{array}$ & Mata atlântica & $\begin{array}{l}\text { Endres et al. } 2005 \\
\text { Endres } \text { et al. } 2007\end{array}$ \\
\hline $\begin{array}{l}\text { Coprophanaeus pertyi } \\
\text { (d'Olsoufieff, 1924) }\end{array}$ & $\begin{array}{c}\text { Áreas abertas em região de brejo de altitude associada a área de } \\
\text { pastagem, bem como em áreas de restinga arbustiva e entre } \\
\text { cerrado e caatinga }\end{array}$ & Lopes et al. 2006; Silva et al. 2007 \\
\hline $\begin{array}{l}\text { Dichotomius geminatus } \\
\quad \text { (Arrow, 1913) }\end{array}$ & Caatinga, tabuleiro e pastagem & $\begin{array}{l}\text { Schiffler et al. } 2003 ; \\
\text { Hernández 2005; Lopes et al. 2006; } \\
\text { Endres et al. } 2007\end{array}$ \\
\hline $\begin{array}{l}\text { Dichotomius semisquamosus } \\
\quad \text { (Curtis, 1845) }\end{array}$ & $\begin{array}{c}\text { Espécie amplamente distribuída em ecossistemas associados a } \\
\text { áreas de pastagem na região nordeste do Brasil, bem como em } \\
\text { áreas degradadas de restinga e mata atlântica }\end{array}$ & $\begin{array}{l}\text { Schiffler } \text { et al. } 2003 \\
\text { Silva } \text { et al. } 2007\end{array}$ \\
\hline $\begin{array}{l}\text { Eurysternus caribaeus } \\
\quad \text { (Herbst, 1789) }\end{array}$ & $\begin{array}{l}\text { Encontrada em cultura de cacau, próximo a remanescente de } \\
\text { mata atlântica }\end{array}$ & Schiffler et al. 2003 \\
\hline $\begin{array}{l}\text { Pseudocanthon xanthurum } \\
\text { (Blanchard, 1843) }\end{array}$ & Área de pastagem, assim como, entre cerrado e caatinga & $\begin{array}{l}\text { Schiffler } \text { et al. } 2003 \text {; } \\
\text { Lopes } \text { et al. } 2006\end{array}$ \\
\hline $\begin{array}{l}\text { Trichillum externepunctatum } \\
\text { Borre, } 1886\end{array}$ & Verificada em um ecótono entre cerrado e caatinga & Lopes et al. 2006 \\
\hline
\end{tabular}

representada por um pequeno número de indivíduos.

Dichotomius aff. sericeus $(\mathrm{N}=3.889)$ e Canthidium $\mathrm{sp} .1$ $(\mathrm{N}=313)$ foram registradas em $100 \%$ das coletas no ambiente de mata e foram as mais abundantes. Já no ambiente aberto, Canthon mutabilis Lucas, $1857(\mathrm{~N}=37)$ e Canthon (Glaphyrocanthon) sp.1 ( $\mathrm{N}=24)$ foram registradas em todas as coletas, sendo também as mais abundantes.

Em todo o estudo foram verificadas 17 espécies para a área aberta, sendo 11 restritas a esse ambiente, enquanto para mata foram registradas 24, sendo 18 espécies restritas. Das espécies coletadas seis (Canthidium sp.1, Canthidium sp. 2, Canthidium sp. 3, Canthon aff. juvencus Harold, 1868, Deltochilum sp.1 e Coprophanaeus cyanescens (d'Olsoufieff, 1924) foram comuns aos dois ambientes. Segundo Saunders et al. (1991), fortes evidências demonstram que as espécies exploram habitats específicos, determinando padrões de distribuição característicos, conforme as condições locais. Deste modo é comum encontrar populações de determinada espécie associadas a um tipo de vegetação, recurso alimentar, microclima, entre outros fatores.

A maioria dos indivíduos (4.417) foram coletados no ambiente de mata, enquanto na área aberta foram registrados apenas 159 espécimes. Grande parte dos indivíduos capturados na mata foi da espécie $D$. aff. sericeus $(\mathrm{N}=3.889)$, o que demonstra uma alta dominância neste ambiente, visto que no ambiente aberto nenhum indivíduo foi coletado. Endres et al. (2007) estudando ecossistema de Mata Atlântica na Paraíba, também verificou uma alta abundância relativa (47\%) para esta espécie neste ecossistema. Segundo Schiffler et al. (2003), esta espécie é típica de Mata Atlântica em localidades de baixa altitude.

No estudo dos índices de diversidade e de equitabilidade das espécies, verificamos para o ambiente de mata os seguintes valores $\left(H^{\prime}=0,81 ; E^{\prime}=0,07\right)$, enquanto para o ambiente aberto $\left(H^{\prime}=3,37 ; E^{\prime}=0,46\right)$. O baixo índice de equitabilidade obtido para o ambiente de mata é reflexo da elevada dominância de Dichotomius aff. sericeus. Como o cálculo da diversidade

Tabela IV. Número e percentuais das espécies de Scarabaeinae (raras e comuns) nos dois ambientes do Refúgio Ecológico Charles Darwin.

\begin{tabular}{lcc}
\hline \multicolumn{1}{c}{ Espécies } & Ambiente aberto & Ambiente de mata \\
\hline Raras "Singletons" & $1(5,88 \%)$ & $6(25 \%)$ \\
Raras "Doubletons" & $5(29,41 \%)$ & $5(20,83 \%)$ \\
Raras (3 - 10 indivíduos) & $6(35,29 \%)$ & $4(16,66 \%)$ \\
Comuns & $5(29,41 \%)$ & $9(37,5 \%)$ \\
Total de espécies & $17(100 \%)$ & $24(100 \%)$ \\
\hline
\end{tabular}


Tabela V. Estimativa da riqueza de espécies para dois ambientes do Refúgio Ecológico Charles Darwin.

\begin{tabular}{lccccc}
\hline \multirow{2}{*}{ Estimadores } & \multicolumn{2}{c}{ Ambiente aberto } & & \multicolumn{2}{c}{ Ambiente de mata } \\
\cline { 2 - 3 } \cline { 5 - 6 } & $\begin{array}{c}\text { Número de } \\
\text { espécies }\end{array}$ & $\begin{array}{c}\text { Desvio } \\
\text { Padrão }\end{array}$ & & $\begin{array}{c}\text { Número de } \\
\text { espécies }\end{array}$ & $\begin{array}{c}\text { Desvio } \\
\text { Padrão }\end{array}$ \\
\hline Chao 1 & 17 & 0,08 & & 26,5 & 2,89 \\
Chao 2 & 18 & 1,58 & & 26,9 & 3,13 \\
Jackknife 1 & 20,33 & 1,66 & & 29,8 & 1,53 \\
Jackknife 2 & 20,86 & 0 & & 31,8 & 0 \\
Espécies & & & & & \\
observadas & 17 & 1,23 & & 24 & 1,86 \\
\hline
\end{tabular}

associa os valores de abundância relativa com o número de espécies, e o índice utilizado nesta análise (Shannon) é mais sensível a mudanças nas espécies raras de uma comunidade, conseqüentemente os índices de diversidade também foram baixos nesse ambiente, uma vez que a proporcionalidade em termos de abundância, entre as espécies desta comunidade é baixa. Segundo Stiling (1999), o máximo de diversidade que pode ser obtida em uma amostra com esse índice é a condição em que todas as espécies possuem abundâncias iguais.

Os dados obtidos pelo uso dos estimadores de riqueza (Chao "1" e "2"; Jackknife "1" e "2") indicaram uma estimativa máxima de riqueza de 21 espécies para o ambiente aberto e 32 espécies para o ambiente de mata (Tab. V). A curva de acumulação de espécies construída baseada em valores de riqueza observada média (Sobs) para os dois ambientes do RECD não apresentou tendência a assíntota (Fig. 1). Quando comparado as curvas obtidas para os dois ambientes podemos perceber que na área aberta houve uma maior tendência a assíntota. O desempenho observado na curva do ambiente de mata reflete o comportamento apresentado pelo grande número de espécies raras nas amostras (espécies singletons, doubletons, uniques e duplicates), as quais são bastante importantes nos cálculos dos estimadores não paramétricos de riqueza (Colwell \& Coddington 1994). Esses resultados apontam para um acréscimo maior no número de espécies nesse ambiente à medida que novos esforços amostrais sejam realizados no RECD.

O estudo da comunidade de escarabeíneos do RECD foi o primeiro registro de levantamento sistematizado de Scarabaeinae com armadilha de interceptação de vôo para o Nordeste Brasileiro, ampliando o registro das espécies para o estado de Pernambuco e contribuindo para o conhecimento da diversidade local destes insetos em remanescente de Mata Atlântica e áreas abertas associadas.

Agradecimentos. A Fundação de Amparo à Ciência e Tecnologia do Estado de Pernambuco - FACEPE (Processo nº06/2003: PPP/MCT/ CNPq/CT-INFRA/FACEPE, de R.C Moura); À CAPES (bolsa de Mestrado de Fernando Silva). A Fernando Z. Vaz-de-Mello, pela identificação de algumas espécies. Aos colegas de laboratório pela colaboração nos trabalhos de campo. A Roberto Siqueira por ter possibilitado o acesso ao RECD.

\section{REFERÊNCIAS}

Andresen, E. 2005. Effects of Season and Vegetation Type on Community Organization of Dung Beetles in a Tropical Dry Forest. Biotropica 37: 291-300.

Brown Jr., K. S. 1991. Conservation of Neotropical environments: Insects as indicators, p. 349-404. In: N. M. Collins \& J. A. Thomas (eds.). The conservation of insects and their habitats. London, Royal Entomological Society/Academic Press.

Brown Jr., K. S. 1997. Diversity, disturbance and sustainable use of Neotropical forests: Insects as indicators for conservation monitoring. Journal of Insect Conservation 1: 25-42.

Brown Jr., K. S. \& A. V. L. Freitas. 2000. Atlantic Forest butterflies: Indicators for landscape conservation. Biotropica 32: 934-956.

Colwell, R. K. 2005. EstimateS: Statistical estimation of species richness and shared species from samples. Version 7.5. User's guide and application published at: http://viceroy.eeb.uconn.edu.

Colwell, R. K. \& J. A. Coddington. 1994. Estimating terrestrial biodiversity through extrapolation. Philosophical Transaction of the Royal Society London series B 345: 101-118.

Costa, C. 2000. Estado de conocimiento de los Coleoptera Neotropicales. In: F. Martin Piera; J. J. Morrone \& A. Melic (eds.). Hacia un Proyecto CYTED para el inventario y estimación de la diversidad Entomológica en Iberoamérica: PrIBES2000. Zaragoza, Sociedad Entomológica Aragonesa (SEA), 326 p.

Dajoz, R. 1978. Ecologia Geral; tradução de Francisco M. Guimarães. Petrópolis, Ed. Vozes, 474 p.

Dale, V.; H. Offerman; R. Frohn \& R. Gardner. 1995. Landscape characterization and biodiversity research, p. 47-66. In: T. J. B. Boyle \& B. Boontawee (eds.). Measuring and monitoring biodiversity in tropical and temperate forest. Bogor, Indonésia, Center for International Forestry Research (CIFOR), 383 p.

Endres, A. A.; A. J. Creão-Duarte \& M. I. M. Hernández. 2007. Diversidade de Scarabaeidae s. str. (Coleoptera) da Reserva Biológica Guaribas, Mamanguape, Paraíba, Brasil: uma comparação entre Mata Atlântica e Tabuleiro Nordestino. Revista Brasileira de Entomologia 51: 67-71.

Endres, A. A.; M. I. M. Hernández \& A. J. Creão-Duarte. 2005. Considerações sobre Coprophanaeus ensifer (Germar) (Coleoptera, Scarabaeidae) em um remanescente de Mata Atlântica no Estado da Paraíba, Brasil. Revista Brasileira de Entomologia 49: 427429.

Escobar, F. 1997. Estudio de la comunidad de coleópteros coprófagos (Scarabaeidae) en un remanente de bosque seco al norte del Tolima. Caldasia 19: 419-430.

Favila, M. E. \& G. Halffter. 1997. The use of indicator groups for measuring biodiversity as related to community structure and function. Acta Zoológica Mexicana 72: 1-25.

Freitas, A. V. L.; I. R. Leal; M. Uehara-Prado \& L. Iannuzzi. 2006. Insetos como indicadores de conservação da paisagem, p. 201225. In: C. F. Rocha; H. Bergalo; M. Van Sluys \& M. A. Alves (orgs.) Biologia da Conservação. Rio de Janeiro, Editora da UERJ.

Gardner, T. A.; M. I. M. Hernández; J. Barlow; C. A. Peres. 2008. Understanding the biodiversity consequences of habitat change: the value of secondary and plantation forests for neotropical dung beetles. Journal of Applied Ecology. Journal compilation - British Ecological Society.

Gimenes, M. R. \& L. dos Anjos. 2003. Efeitos da fragmentação florestal sobre as comunidades de aves. Acta Scientiarum. Biological Sciences 25: 391-402.

Halffter, G. 1991. Historical and ecological factors determining the geographical distribution of beetles (Coleoptera: Scarabaeidae: Scarabaeinae). Folia Entomológica Mexicana 82: 195-238.

Halffter, G. \& L. Arellano. 2002. Response of dung beetle diversity to human induced changes in a tropical landscape. Biotropica 34 : $144-154$.

Halffter, G. \& M. E. Favila. 1993. The Scarabaeinae an Animal Group for Analysing, inventorying and Monitoring Biodiversity in 
Tropical Rainforest and Modified Landscapes. Biology International 27: 15-21.

Halffter, G. \& E. G. Matthews. 1966. The Natural History of Dung Beetles of the Subfamily Scarabaeinae (Coleoptera, Scarabaeidae). Folia Entomologica Mexicana 12-14: 1-312.

Hanski, I. \& Y. Cambefort. 1991. Dung beetles ecology. New Jersey, Princeton University Press, $481 \mathrm{p}$.

Hernández, M. I. M. 2005. Artrópodes: Besouros Scarabaeidae (Coleoptera) do Curimataú, Paraíba, Brasil, p. 369-380. In: F. S. Araújo, M. J. N. Rodal \& M. R. V. Barbosa (eds.). Análise das Variações da Biodiversidade do Bioma Caatinga para Suporte a Estratégias Regionais de Conservação. Brasília, PROBIO - Ministério do Meio Ambiente, 434 p.

Hernández, M. I. M. 2007. Besouros escarabeíneos (Coleoptera: Scarabaeidae) da Caatinga Paraibana, Brasil. Oecologia brasiliensis 11: $356-364$.

Howden, H. F. \& V. G. Nealis. 1975. Effects of clearing in a tropical rain forest on the composition of coprophagous scarab beetle fauna (Coleoptera). Biotropica 7: 77-83.

Kenney, A. J. \& C. J. Krebs. 2000. Programs for Ecological Methodology. Vancouver, University of British Columbia press, $620 \mathrm{p}$.

Klein, B. C. 1989. Effects of forest fragmentation on dung and carrion beetle communities in central Amazonia. Ecology 70: 1715-1725.

Lawrence, J. F. \& E. B. Britton. 1991. Coleoptera (beetles), p. 543683. In: I. Naumann (ed.). The Insects of Australia: A textbook for students and research workers (CSIRO). New York, Cornell University Press, $1137 \mathrm{p}$.

Lawrence, J. F. \& A. F. Newton Jr. 1995. Families and subfamilies of Coleoptera (with selected genera, notes, references and data on family-group names), p. 779-1006. In: J. F. Pakaluk \& S. A. Slipinski (eds.). Biology, Phylogeny and classification of Coleoptera: Papers celebrating the 80th birthday of Roy A. Crowson.. Warszawa, Muzeum i Instytut Zoologii PAN, 1092 p.

Lopes, P. P.; J. N. C. Louzada \& F. Z. Vaz-De-Mello. 2006. Organization of dung beetle communities (Coleoptera, Scarabaeidae) in areas of vegetation re-establishment in Feira de Santana, Bahia, Brazil. Sitientibus Série Ciências Biológicas 6: 261-266.

Louzada, J. N. C. \& F. S. A. Lopes. 1997. A comunidade de Scarabaeidae copro-necrófagos (Coleoptera) de um fragmento de Mata Atlântica. Revista Brasileira de Entomologia 41: 117-121.

Myers, N.; R. A. Mittermeier; C. G. Mittermeier; G. B. Fonseca \& J. Kents. 2000. Biodiversity hotspots for conservation priorities. Nature 403: 853-858.

Nichols, E.; T. Larsen; S. Spector; A. L. Davis; F. Escobar; M. Favila; K. Vulinec; The Scarabaeinae Research Network. 2007. Global dung beetle response to tropical forest modification and fragmentation: A quantitative literature review and meta-analysis. Biological Conservation 137: 1-19.

Primack, R. B. \& E. Rodrigues. 2001. Biologia da Conservação. Londrina, Ed. Planta, 328 p.

Ranta, P.; T. Blom; J. Niemela; E. Joensuu \& M. Siitonen. 1998. The fragmented atlantic rain forest of Brasil: size, shape and distribution of forest fragments. Biodiversity Conservation 7: 385-403.

Rodrigues, S. R. \& L. C. Marchini. 2000. Ocorrência de besouros coprófagos em dois diferentes ambientes. Revista Brasileira de Entomologia 44: 35-38.

Santiago, A. C. P. \& I. C. L. Barros. 2003. Pteridoflora do Refúgio Ecológico Charles Darwin (Igarassu, Pernambuco, Brasil). Acta Botânica Brasileira 17: 597-604.

Saunders, D. A.; R. J. Hobbs \& C. R. Margules. 1991. Biological consequences of ecosystem fragmentation: a review. Conservation Biology 5: 18-34.

Schiffler, G.; F. Z. Vaz-De-Mello \& C. O. Azevedo. 2003. Scarabaeidae s. str. (Coleoptera) do Delta do Rio Doce e Vale do Suruaca no município de Linhares, estado do Espírito Santo, Brasil. Revista Brasileira de Zoociências 5: 205-211.

Silva, F. A. B.; M. I. M. Hernández; S. Ide \& R. C. Moura. 2007. Comunidade de escarabeíneos (Coleoptera, Scarabaeidae) copronecrófagos da região de Brejo Novo, Caruaru, Pernambuco, Brasil. Revista Brasileira de Entomologia 51: 228-233.

Spector, S. \& S. Ayzama. 2003. Rapid turnover and edge effects in Dung Beetle assemblages (Scarabaeidae) at a Bolivian Neotropical Forest-Savanna Ecotone. Biotropica 35: 394-404.

Statsoft. 1998. Statistica for Windows. Tulsa: StatSoft.

Stiling, P. 1999. Ecology. Theories and Applications. New Jersey, Pratice Hall, 638 p.

Tabarelli, M.; M. D. V. C. Melo \& O. C. Lira. 2006. Nordeste e Estados do Nordeste, menos Sergipe, p. 149-164. In: M. Campanili \& M. Prochnow (eds.). Mata Atlântica: Uma rede pela floresta. Brasília, Rede de ONGs da Mata Atlântica (RMA), 332 p.

Thomazini, M. J. \& A. P. B. W. Thomazini. 2000. A fragmentação florestal e a diversidade de insetos nas florestas tropicais úmidas. Rio Branco, Embrapa Acre (Embrapa Acre. Documentos, 57), $21 \mathrm{p}$.

Vaz-de-Mello, F. Z. 2000. Estado atual de conhecimento dos Scarabaeidae s. str. (Coleoptera: Scarabaeoidea) do Brasil, p. 183195. In: F. Martin Piera; J. J. Morrone \& A. Melic (eds.). Hacia un Proyecto CYTED para el inventario y estimación de la diversidad Entomológica en Iberoamérica: PrIBES-2000. Zaragoza, Sociedad Entomológica Aragonesa (SEA), 326 p.

Recebido em 26/12/2007; aceito em 26/09/2008 